\title{
Prospecção tecnológica em bases de patentes com foco em corantes e pigmentos alimentícios obtidos de fontes naturais
}

\author{
Technological prospecting on patent bases focusing on food dyes and pigments obtained from \\ natural sources \\ Prospección tecnológica en base de patentes centrada en colorantes y pigmentos alimentarios \\ obtenidos de fuentes naturales
}

Recebido: 06/03/2021 | Revisado: 14/03/2021 | Aceito: 18/03/2021 | Publicado: 26/03/2021

\author{
Mayane Santana Vieira Santos \\ ORCID: https://orcid.org/0000-0003-0262-1911 \\ Universidade Federal de Sergipe, Brasil \\ E-mail: mayanevss@gmail.com \\ Débora Eloá Lima Santos \\ ORCID: https://orcid.org/0000-0002-7999-4567 \\ Universidade Federal de Sergipe, Brasil \\ E-mail: deebora.eloa@gmail.com \\ Jane de Jesus da Silveira Moreira \\ ORCID: https://orcid.org/0000-0001-7586-4856 \\ Universidade Federal de Sergipe, Brasil \\ E-mail: jjsm.ufs@gmail.com \\ Marcio Nannini da Silva Florêncio \\ ORCID: https://orcid.org/0000-0001-5557-4181 \\ Universidade Federal de Sergipe, Brasil \\ E-mail: marcio_nannini@hotmail.com \\ Antonio Martins de Oliveira Junior \\ ORCID: https://orcid.org/0000-0002-8635-7048 \\ Universidade Federal de Sergipe, Brasil \\ E-mail: amartins.junior@gmail.com
}

\begin{abstract}
Resumo
A indústria alimentícia tem sido desafiada a buscar alternativas naturais e saudáveis para seus produtos, sem prejudicar sua segurança e principais características. Neste contexto, os corantes naturais surgem como uma alternativa à substituição dos corantes sintéticos no segmento alimentar. Como consequência dos diversos estudos e inovações, a proteção patentária se mostra como uma ferramenta de proteção para o inventor. Este trabalho teve como objetivo explorar as tecnologias patenteadas a respeito de corantes e pigmentos alimentícios obtidos a partir de fontes naturais e renováveis, a fim de identificar os avanços tecnológicos e tendências mercadológicas no cenário nacional e internacional. Foi realizado um mapeamento tecnológico a partir de documentos de patentes, utilizando o software de busca e análise, ORBIT da empresa Questel e a base de dados brasileira do INPI. Foi possível constatar o crescimento dos depósitos, destacando-se os incentivos à pesquisa, desenvolvimento e inovação. A China foi o principal país que depositou patentes. Observou-se que tanto no nacional quanto internacionalmente, as principais partes interessadas em proteger suas tecnologias eram empresas privadas, sendo a Chr. Hansen a empresa com o maior número de patentes em seu portfólio. O campo da tecnologia relaciona-se principalmente as áreas da indústria de alimentos, química de materiais e indústria farmacêutica.
\end{abstract}

Palavras-chave: Inovação; Indústria alimentícia; Tecnologia.

\begin{abstract}
The food industry has been challenged to find natural and healthy alternatives in the development of new products, without changing its safety and main characteristics. In this context, natural dyes appear as an alternative to the substitution of synthetic dyes in the food segment. Patent protection is shown to be a protection tool for inventors as a result of various research and innovations. This paper aimed to explore patent technologies regarding food dyes and pigments obtained from natural and renewable sources, in order to identify technological advances and market trends in the national and international scenario. A technological mapping was carried out based on patent bases, using the search and analysis software, ORBIT from Questel and the Brazilian INPI database. It was possible to evidence a growth in deposits, highlighting the incentives for research, development and innovation. China was the main country that filed for patents. It was observed that both nationally and internationally, the main interested parties in protecting their technologies were private companies, being CHR. HANSEN the company with the highest number of patents in
\end{abstract}


its portfolio. The field of technology relates mainly to the areas of the food industry, materials chemistry and the pharmaceutical industry.

Keywords: Innovation; Food industry; Technology.

\section{Resumen}

La industria alimentaria se ha visto desafiada a buscar alternativas naturales y saludables para sus productos, sin comprometer su seguridad y principales características. En este contexto, los tintes naturales aparecen como una alternativa a la sustitución de los tintes sintéticos en el segmento alimentario. Como consecuencia de los diversos estudios e innovaciones, la protección mediante patente se muestra como una herramienta de protección para el inventor. Este trabajo tuvo como objetivo explorar las tecnologías patentadas sobre colorantes y pigmentos alimentarios obtenidos de fuentes naturales y renovables, con el fin de identificar los avances tecnológicos y las tendencias del mercado en el escenario nacional e internacional. Se realizó un mapeo tecnológico a partir de documentos de patente, utilizando el software de búsqueda y análisis ORBIT de la empresa Questel y la base de datos brasileña del INPI. Se pudo constatar el crecimiento de los depósitos, destacando los incentivos para la investigación, el desarrollo y la innovación. China fue el principal país que presentó patentes. Se observó que, tanto a nivel nacional como internacional, las principales partes interesadas en proteger sus tecnologías fueron las empresas privadas, siendo Chr. Hansen la empresa con mayor número de patentes en su portafolio. El campo de la tecnología se relaciona principalmente con las áreas de la industria alimentaria, la química de materiales y la industria farmacéutica. Palabras clave: Innovación; Industria alimentaria, Tecnología.

\section{Introdução}

A cor é um dos primeiros atributos utilizado como critério para compra e escolha de alimentos, devido à influência na sensação de outras características sensoriais como aroma, sabor e textura (Costa \& Vendruscolo, 2017). Na indústria de alimentos, os corantes são usados como aditivos com a finalidade de conferir, intensificar ou padronizar a coloração, podendo restaurar a aparência original dos produtos alimentícios afetada durante as etapas de processamento, armazenamento, embalagem e distribuição (Oliveira et al., 2019).

Segundo a ANVISA, aditivo alimentar é qualquer ingrediente adicionado intencionalmente aos alimentos, sem propósito de nutrir, com o objetivo de modificar as características físicas, químicas, biológicas ou sensoriais, durante qualquer etapa, desde a fabricação à distribuição e consumo (Brasil, 1997).

Apesar da importância do uso dos aditivos, é evidente a mudança nos hábitos alimentares por parte dos consumidores, que passaram a se preocupar com a qualidade dos alimentos consumidos, buscando por produtos naturais, saudáveis e práticos. Tal comportamento representa um desafio para a indústria alimentícia que tem pesquisado alternativas à substituição dos aditivos sintéticos por naturais, sem prejudicar a segurança alimentar e que mantenham a eficiência quanto à manutenção das características dos produtos ao longo do tempo (Dall'Agnol et al., 2013). Nesse sentido foi observado um aumento nas pesquisas sobre aditivos naturais, provenientes da fauna e da flora.

No setor industrial, a inovação é uma questão de sobrevivência e, portanto, deve ser considerada na tomada de decisões. Para determinar o nível de inovação, são realizados estudos prospectivos. Os Estudos de Prospecção Tecnológica, também chamados de estudos de futuro, ou forecast (ing), foresight (ing) ou future studies, fornecem as principais tendências no contexto mundial, sendo possível classificar essas tecnologias por setor da economia (Borschiver, 2008).

Esses estudos são realizados em banco de dados patentários permitindo que o pesquisador entenda sua tecnologia, identifique pontos fortes e fracos do seu invento e reconheça um diferencial realmente inédito. Além disso, servem como ferramenta para entender a evolução tecnológica em determinado setor, entender a evolução tecnológica ao longo dos anos, as perspectivas mercadológicas, possibilitando que empresas melhorem seus processos produtivos impulsionados por outros métodos já existentes e desenvolvam novas tecnologias com base nas atuais tendências (Ghesti et al., 2017).

Em nível nacional os depósitos de patentes são feitos na base de dados do Instituto Nacional de Propriedade Industrial (INPI). O mesmo, é um órgão pertencente à federação brasileira, vinculado ao Ministério do Desenvolvimento, Indústria e Comércio Exterior (Comissão Permanente de Propriedade Intelectual, n.d). 
Outra possibilidade é o acesso a dados de patentes usando ferramentas de busca, como o ORBIT®, um software que permite acesso à base de dados da FAMPAT, possui um sistema de busca, seleção, análise e exportação de informações contidas em documentos patentários, produzido por uma empesa franco-americana, a Questel. A cobertura geográfica dessa base compreende publicações de quase uma centena de países e autoridades de patentes, incluindo pedidos referente à mesma invenção depositados em diferentes países, dessa forma evitando a duplicidade dos dados gerando resultados mais específicos para o estudo prospectivo (Axonal, 2016; Barros et al., 2020).

Diante do exposto, este trabalho teve como objetivo apresentar a prospecção tecnológica de patentes sobre os corantes e pigmentos alimentícios obtidos a partir de fontes naturais e renováveis, a fim de verificar os avanços tecnológicos e tendências mercadológicas em nível nacional e internacional.

\section{Metodologia}

O presente estudo emprega pesquisa documental visto que foca no mapeamento de dados patentários (Ribeiro, 2018) e possui uma abordagem quali-quanti, na qual os resultados numéricos do número total de documentos gerados são complementados com a descrição dos dados coletados que possuem caráter qualitativo. Para Pereira et al. (2018) em métodos quantitativos utiliza-se a coleta de dados quantitativos ou numéricos por meio de medições de grandezas, gerando conjuntos de dados que podem ser analisados por meio de técnicas matemáticas, enquanto os métodos qualitativos são aqueles nos quais é importante a interpretação por parte do pesquisador com suas opiniões sobre o fenômeno em estudo. Quando um método complementa o outro, este é classificado como um método quali-quanti.

Foi realizado um mapeamento patentário. Segundo Ribeiro (2018), é uma técnica que possibilita identificar os inventores, os titulares, os tipos de tecnologias, as referências a patentes e artigos anteriores, ajudando a identificar os detentores da tecnologia. Para isso, realizou-se um levantamento de dados referente às tecnologias empregando corantes e pigmentos naturais em alimentos, em âmbito nacional e mundial, utilizando-se dos recursos das bases do INPI (www.inpi.gov.br) e o software ORBIT® (www.orbit.com) que dá acesso a patentes na base da FAMPAT.

A estratégia de busca utilizada para coletar os dados desta pesquisa foi baseada no estudo realizado por Barros et al. (2020) com adaptações. O termo de busca utilizado no ORBIT® foi ((natural w (pigment or dye)) and food)/TI/AB AND (A23+)/IPC. Utilizou-se o operador booleano "AND" e "W", o caractere de truncamento "+" e os parênteses "()", que servem para refinar a busca pretendida. O operador booleano "W" busca por termos contíguos, exatamente na ordem digitada, o operador "AND" é usado para encontrar ambos os termos de busca presentes. O caractere de truncamento "+" permite deixar em aberto qualquer número de caracteres, o uso de parênteses "()" determina a ordem do processamento da pesquisa. O termo TI refere-se à busca dos termos no título da patente, enquanto o termo $\mathrm{AB}$, no resumo (Fernandes et al. 2017).

No INPI, a estratégia de busca empregada utilizou a Classificação Internacional de Patentes (IPC, da sigla em inglês), visando otimizar a busca por meio de suas áreas tecnológicas. Em cada área, há grupos principais e subgrupos, hierarquizando as tecnologias. O uso dessa classificação determina o segmento tecnológico ao qual uma patente pertence, facilitando a busca e definindo o nicho da tecnologia. (Fernandes et al. 2017) Foram utilizadas as o subgrupo "C09B 61/00" que trata de "corantes de origem natural preparados a partir de fontes naturais" e o grupo principal "A23" que se refere a "alimentos ou gêneros alimentícios; seu tratamento não coberto por outras classes".

As buscas foram realizadas nos meses de setembro e outubro de 2020 e investigou o número total de depósitos, o país de origem dos depositantes, os principais depositantes, o status legal e a situação do pedido. 


\section{Resultados e Discussão}

Com a realização da busca nas bases citadas, foram encontrados 187 depósitos na base da FAMPAT e 31 no INPI. Na Figura 1, pode-se observar a evolução dos mesmos ao longo dos anos. Ao analisar os dados nas bases estudadas, verificou-se que o primeiro depósito ocorreu em 1977 na FAMPAT. A partir de 1999, em todos os anos posteriores houve pedidos de patente, sendo 1997, 2014 e 2015 os anos com maior número de depósitos. No INPI o primeiro depósito foi realizado por um cidadão norueguês no ano de 1989. Novos pedidos voltaram a ser depositados apenas em 1997, sendo um de Israel e dois do Brasil.

Figura 1. Evolução dos pedidos de patente envolvendo o uso de corantes naturais alimentícios de acordo com as bases INPI e FAMPAT.

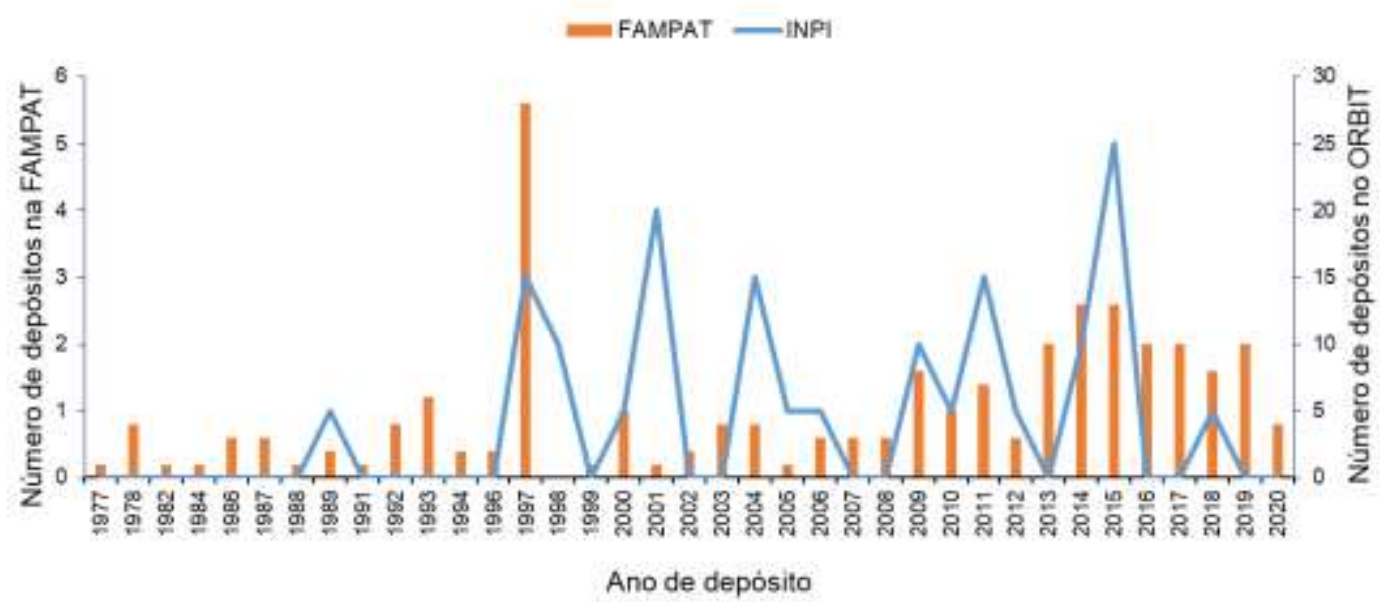

Fonte: Autores (2020).

O hábito de utilizar corantes extraídos de fontes naturais remete-nos as civilizações antigas. Porém, com a descoberta dos corantes sintéticos nos séculos XVIII e XIX, cresceu o interesse industrial por esses proporcionarem melhoria na aparência dos produtos e consequente aceitação dos consumidores (Food Ingredients Brasil, 2009).

Na década passada, esse cenário sofreu mais uma modificação, reflexo dos acontecimentos e tendências em que se verifica a procura por produtos naturais e "clean label". Isso incentivou os investimentos em estudos e pesquisas para melhorar a estabilidade, métodos de extração e outros fatores que inviabilizavam o uso do corante natural na indústria de alimentos (Prado, 2003).

Analisando a última década, percebe-se um crescimento de depósitos até a metade da mesma, evidenciando os incentivos à pesquisa, desenvolvimento e inovação. De 2015 a 2020 têm-se um decréscimo. Nesse mesmo período, o Brasil enfrentava uma crise econômica tendo início no ano de 2014 com alta desvalorização cambial, setor industrial com baixa demanda interna e externa, somado à baixa produtividade e competitividade. Até março de 2015, a produção industrial brasileira alcançou uma margem de queda de 5,9\%, o setor de bens de capital caiu 18\%, sinalizando menores investimentos (Empresa de Pesquisa Energética, 2015).

Analisando os principais países depositantes, observa-se que, no âmbito internacional (Figura 2), a China efetuou $46 \%$ dos depósitos de patentes sobre a tecnologia em estudo, seguida da República da Coreia (KR) com 16\% e Japão com 15,5\%. É possível notar que tal tecnologia encontra-se concentrada em países asiáticos, o atual crescimento econômico destes também pode atuar como fator de contribuição para o desenvolvimento de novas tecnologias e posteriores patentes.

É importante ressaltar que o perfil de depósitos é similar em ambas as bases e não existem depósitos de patentes referentes a corantes naturais realizados pelo Brasil na base de dados da FAMPAT. Além dos países asiáticos, nota-se um 
pequeno número de depósitos realizados pelos escritórios europeu (EP) e internacional (WO) com 2,7\% e 1,1\%, respectivamente.

Figura 2. Principais países depositantes das patentes envolvendo uso de corantes naturais alimentícios na base da FAMPAT.

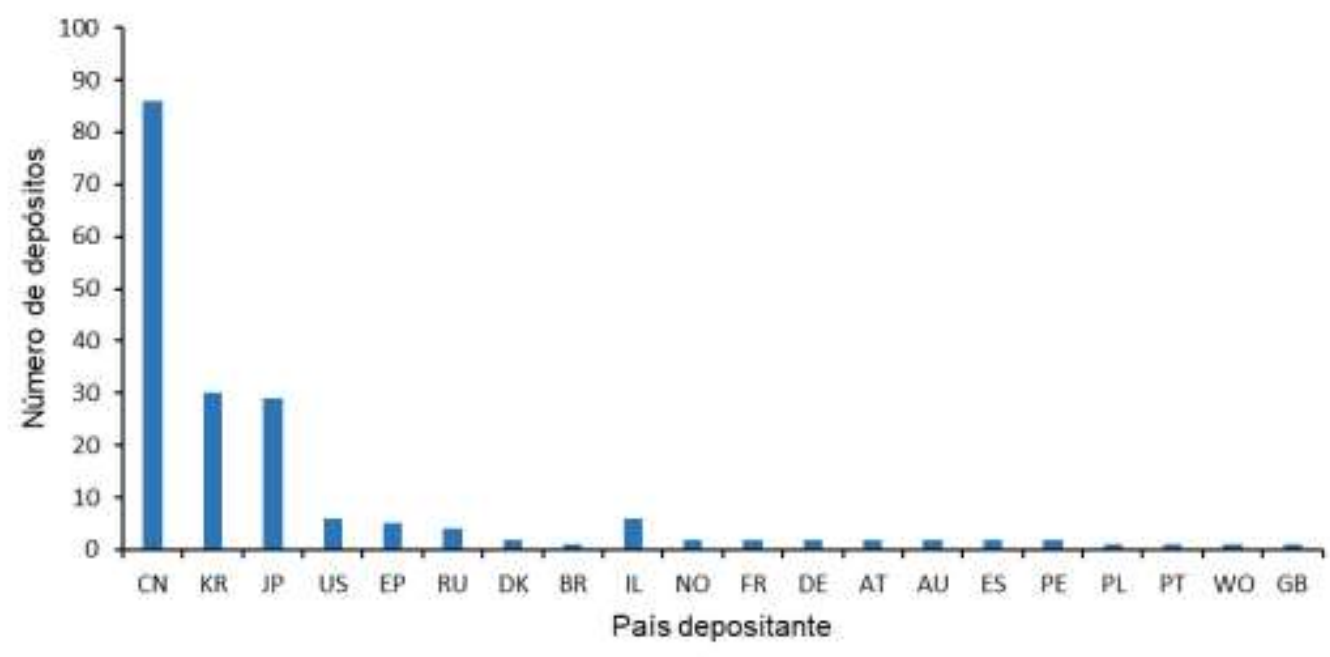

Fonte: Autores (2020).

No NPI (Figura 3), nota-se que a maioria dos depósitos foram realizados pelo escritório europeu de patentes (EP) com 42\%, o Brasil aparece com 25,8\% dos depósitos, seguido dos Estados Unidos (US) com 16,1\%.

Nos Estados Unidos, é permitido o uso de apenas 12 corantes sintéticos, contra 36 naturais certificados pela Food and Drug Administration (FDA). Essa restrição tende a aumentar proporcionando a aplicação de novas alternativas de corantes extraídos de fontes naturais que possam ser utilizados na indústria de alimentos (FDA, 2015).

Figura 3. Principais países depositantes das patentes envolvendo o uso de corantes naturais alimentícios na base do INPI.

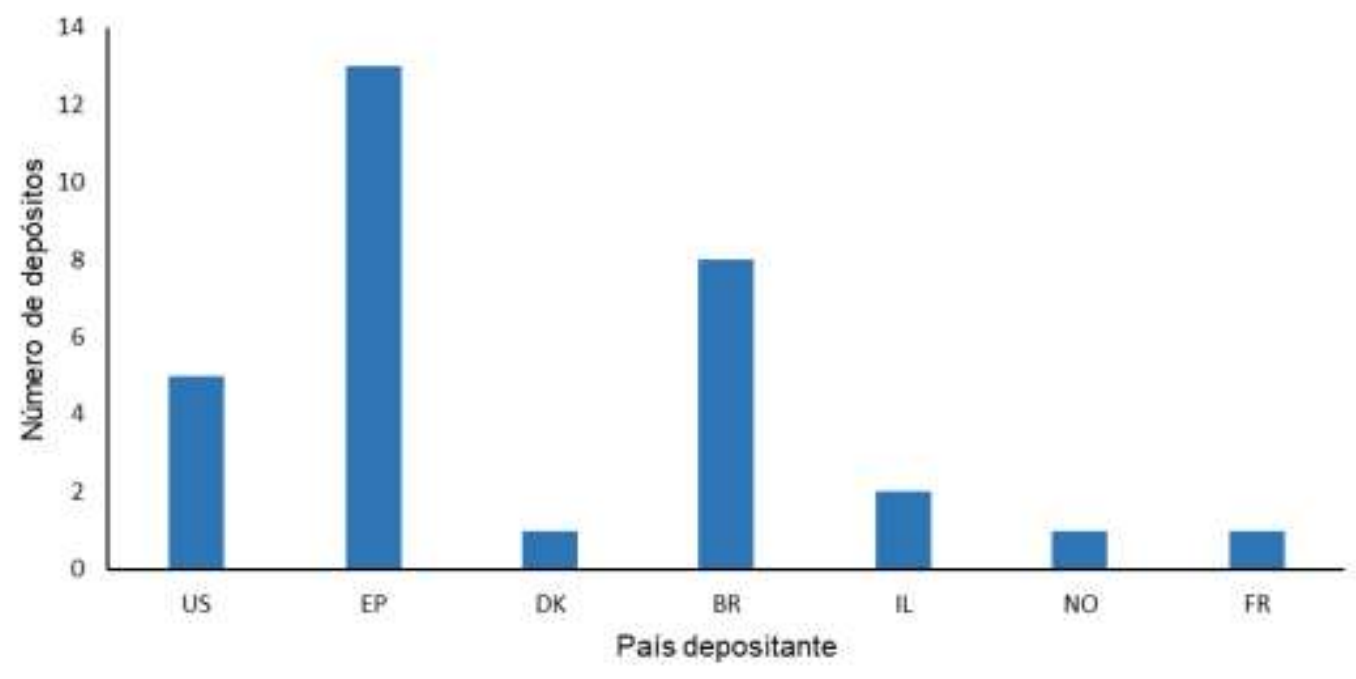

Fonte: Autores (2020). 
Com a crescente demanda de corantes e pigmentos naturais por parte dos consumidores, estima-se que até 2025 o mercado mundial de corantes naturais arrecade 37,49 bilhões de dólares, devido à procura por alimentos que ofereçam propriedades funcionais e à rápida expansão do setor de alimentos e bebidas em todo o mundo. (Grand View Research, 2017).

A análise das classes dos principais depositantes é ilustrada na Figura 4. Pode-se perceber que mais da metade dos depósitos foram realizados por empresas privadas em ambas as bases estudadas. Os Institutos de Ciência e Tecnologia (ICTs) aparecem com $27 \%$ e $21 \%$ na FAMPAT e INPI, respectivamente.

Figura 4. Classes dos principais depositantes das patentes sobre o uso de corantes naturais alimentícios. (a) FAMPAT, (b) INPI.

(a)

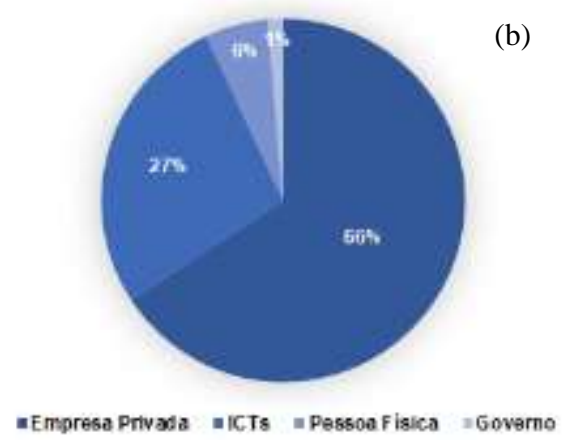

(b)

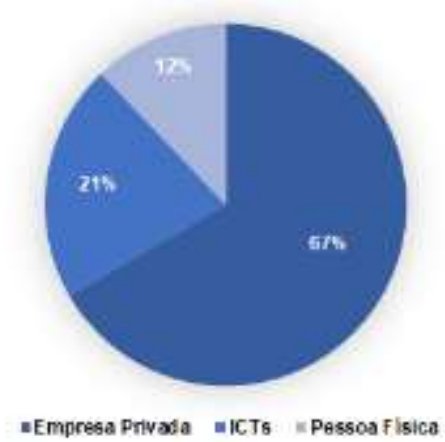

Fonte: Autores (2020).

Meinicke (2008) aponta a Chr. Hansen (Dinamarca), Warner-Jenkinson Europe LTD. (Reino Unido), Kalsec Inc. (Estados Unidos) e Quest Internacional (Países Baixos) como líderes no mercado internacional de corantes naturais.

As Figuras 5 e 6 ilustram a quantidade de depósitos feitos pelos principais depositantes nas bases da FAMPAT e INPI, respectivamente. Observa-se que a Chr. Hansen lidera com 15\% na FAMPAT e 19,4\% no INPI. Outra empresa que se destaca é a Lycored Poducts Industries LTD. com 9,7\% dos depósitos no INPI. Apesar de grandes corporações se destacarem, alguns ICTs possuem documentos patentários depositados, como é o caso das Universidades Federais de São Paulo, Pará, Paraná e Lavras, que aparecem com 1 depósito cada na base do INPI.

Figura 5. Principais depositantes das patentes sobre o uso de corantes naturais alimentícios na base da FAMPAT

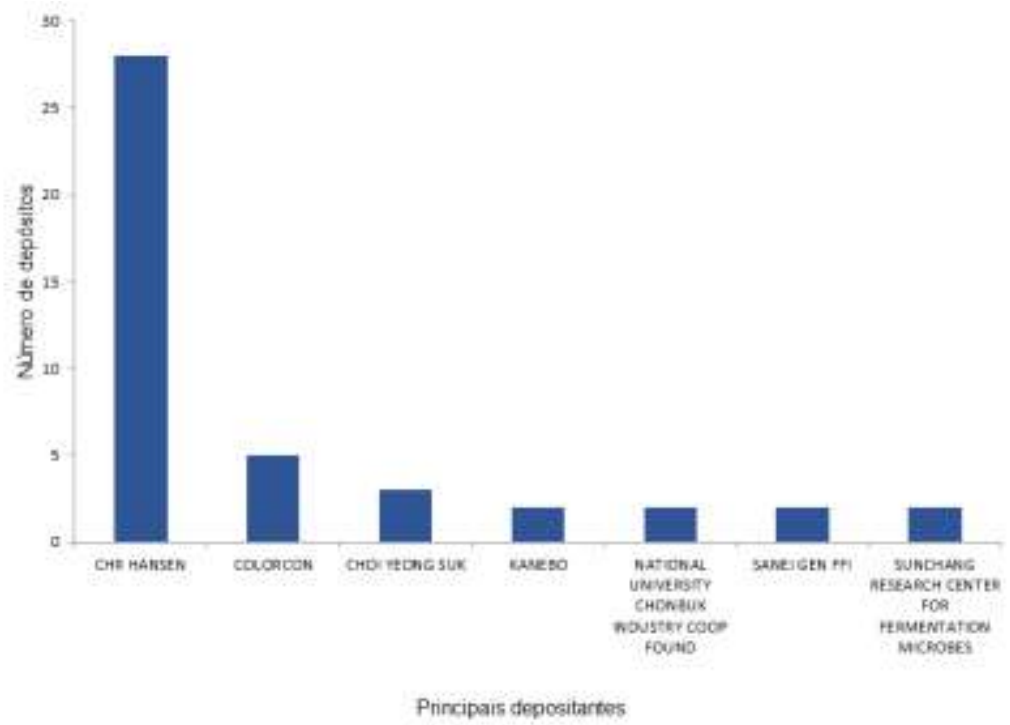

Fonte: Autores (2020). 
A mudança na política de inovação do Brasil, impulsionada pelo Núcleo de Inovação Tecnológica (NIT) e pela Lei da Inovação (Brasil, 2016), estimulou Institutos de Ciência e Tecnologia a promoverem proteção das tecnologias desenvolvidas, havendo maior engajamento com empresas, o que resultou no aumento anual do número de universidades depositantes.

Figura 6. Principais depositantes das patentes envolvendo o uso de corantes naturais alimentícios na base do INPI

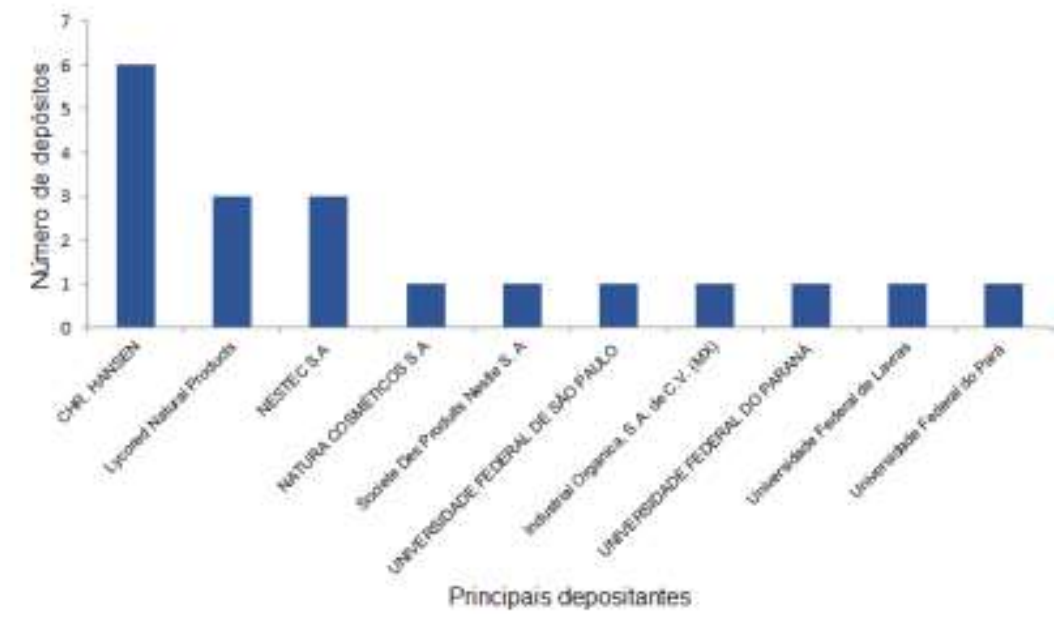

Fonte: Autores (2020).

Em relação ao status legal dos pedidos, a maioria na base de dados da FAMPAT (Figura 7a) encontra-se inativo, apesar de existir muito investimento na tecnologia em alguns países, como China. No Brasil (Figura 7b), 42\% dos pedidos estão ativos, porém aqueles realizados por residentes encontram-se caducados ou indeferidos, sugerindo falta de investimento na tecnologia.

Figura 7. Status legal dos pedidos de patentes sobre o uso de corantes/ pigmentos naturais. (a) FAMPAT, (b) INPI.

(a)

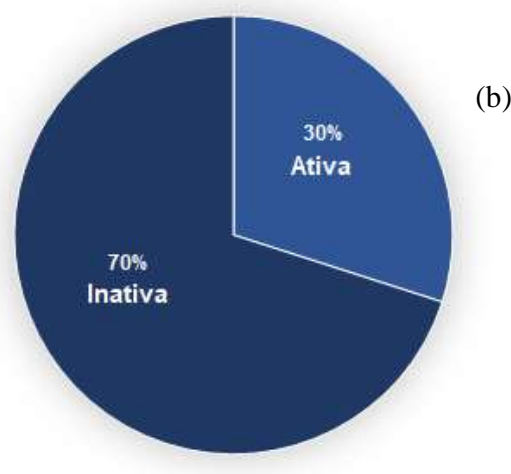

(b)

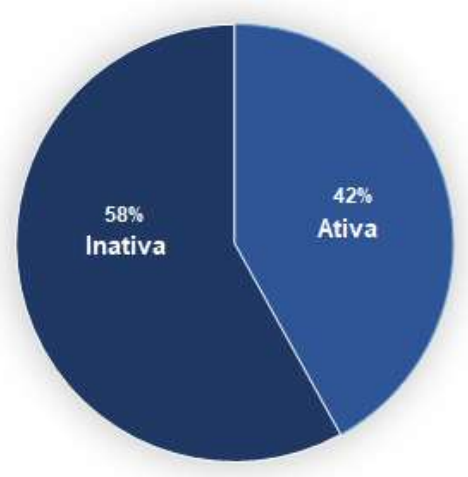

Fonte: Autores (2020).

Ao analisar a situação dos pedidos, percebe-se que em nível nacional (Figura 8a), a maioria estão caducados por falta de pagamento ou pendentes aguardando análise, ambos com mesma porcentagem (36\%), revogados $19 \%$ e apenas 3\% concedidos. Na da FAMPAT (Figura 8b), nota-se que 42\% apresentam caducidade, ou seja, a tecnologia possui livre acesso, $16 \%$ aguarda análise, $13 \%$ ganhou concessão e 15\% tiveram suas patentes expiradas, perdendo a proteção. 
Figura 8. Situação dos pedidos de patentes sobre o uso de corantes/pigmentos naturais. (a) INPI, (b) FAMPAT.
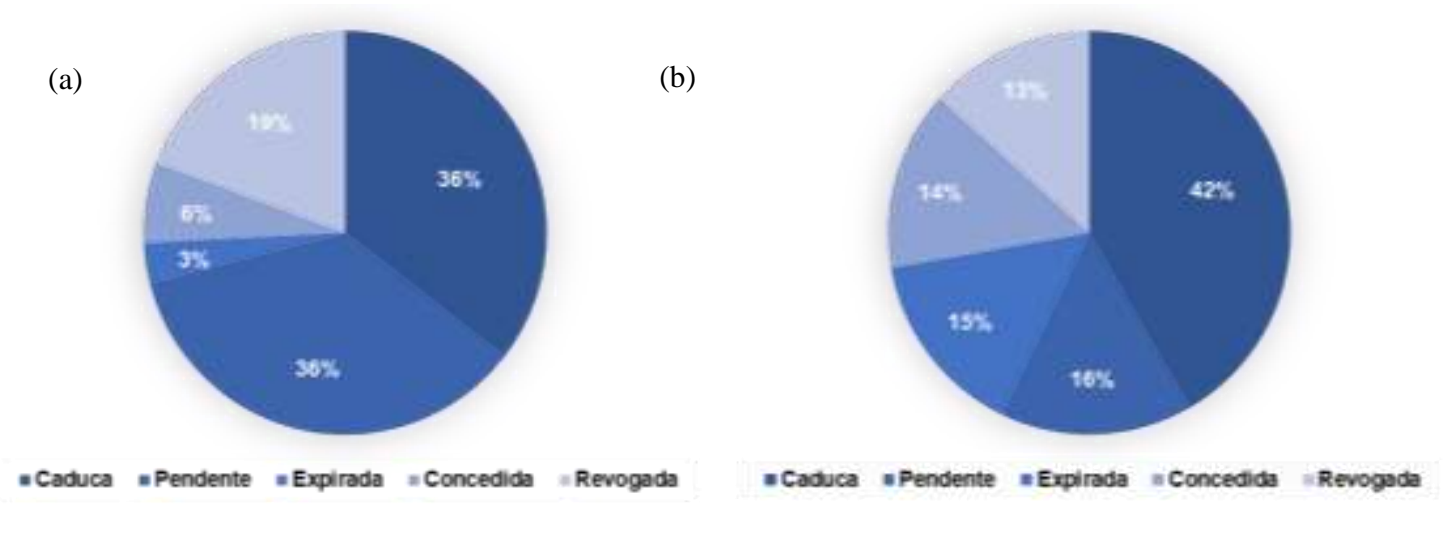

Fonte: Autores (2020).

Na Figura 9 pode-se observar a distribuição das principais áreas tecnológicas de aplicação dos corantes/pigmentos naturais. Dos 187 pedidos de patentes analisados na FAMPAT, 56\% relacionam-se com a química alimentar, 18\% à química dos materiais, $11 \%$ à indústria farmacêutica e outras áreas não especificadas e $3 \%$ à química orgânica.

Figura 9. Domínio da tecnologia dos pedidos de patentes sobre o uso de corantes/pigmentos naturais.

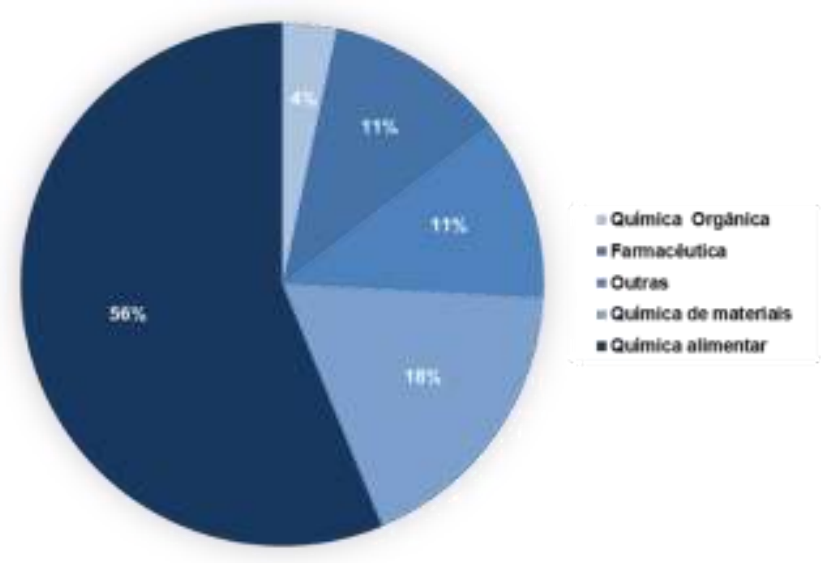

Fonte: Autores (2020).

Há uma variedade de produtos no mercado que utilizam corantes naturais em sua formulação, a exemplo de bebidas, molhos, sopas, sorvetes, massas, entre outros, porém nem sempre o uso desses aditivos é viável devido à diversos parâmetros que afetam sua estabilidade, mas ao mesmo tempo pesquisas e estudos têm permitido o desenvolvimento de técnicas que podem torná-los mais estáveis, viabilizando e ampliando suas aplicações (Rêgo et al., 2015).

\section{Conclusão}

Os corantes sintéticos continuam sendo os mais utilizados pela indústria alimentícia, quando comparados com os naturais. Entretanto, o segmento de corantes naturais vem crescendo devido à percepção dos seus benefícios por parte dos consumidores. 
Os resultados deste estudo demonstram que tanto em bases de dados internacional quanto nacional a proteção patentária na área de corantes e pigmentos alimentícios é muito explorada e que está em constante expansão indicando que muitas inovações têm sido realizadas, principalmente por países asiáticos como China, Japão e Coréia do Sul.

No âmbito nacional, o Brasil apresenta depósito de $1 \frac{1}{4}$ das patentes, apesar de ser um grande mercado para os corantes e um país promissor na obtenção de corantes naturais de diversas fontes, devido à sua grande biodiversidade.

Para trabalhos futuros, sugere-se a ampliação da abordagem em outras bases de patentes, a fim de obter uma abrangência maior. Além disso, a investigação das produções científicas sobre a tecnologia abordada trará resultados interessantes a respeito da cooperação entre os agentes de inovação envolvidos na geração e na proteção da tecnologia.

\section{Referências}

Axonal Consultoria Tecnológica, \& Suzuki, H. (2016). Orbit.com: Visão Geral Sobre o Sistema. Axonal. https://axonal.com.br/arquivos/PDF/Orbit_Visao_Geral_Sistema_PARTES_1_a_3_BUSCA_VISUALIZACAO_SELECAO.pdf.

Barros, G. M., Anjos, M. S. dos \& Barros, G. M. (2020). Prospecção tecnológica do pequizeiro. Research, Society and Development, 9(9), 1-15. http://dx.doi.org/10.33448/rsd-v9i9.7957.

Brasil. Lei $n^{\circ}$ 13.243/2016, de 11 de janeiro de 2016 (2016). Dispõe sobre estímulos ao desenvolvimento científico, à pesquisa, à capacitação científica e tecnológica e à inovação... Brasília. 2016. http://www.planalto.gov.br/ccivil_03/_ato2015-2018/2016/lei/113243.html.

Brasil. Portaria 540, de 27 de outubro de 1997. (1997). Aprova o Regulamento Técnico: Aditivos alimentares - definições, classificação e emprego. Brasil. http://bvsms.saude.gov.br/bvs/saudelegis/svs1/1997/prt0540_27_10_1997.html.

Borschiver, S. (2008). Apostila de Curso de Pós-Graduação. UFRJ.

Costa, J. P. V. \& Vendruscolo, F. (2017). Production of red pigments by Monascus ruber CCT 3802 using lactose as a substrate. Biocatalysis and Agricultural Biotechnology, 11, 50-55. https://doi.org/10.1016/j.bcab.2017.05.010.

Dall'Agnol, R. P., Santos, J. A. B., Machado, G. J. C., Silva, R., \& Santos, A. P. S. (2013). A utilização de corantes artificiais em produtos alimentícios no Brasil. Anais Do Simpósio Internacional De Inovação Tecnológica - SIMTEC, 1(1), 26-37. https://doi.org/10.7198/s2318-34032013001003.

Empresa de Pesquisa Energética. (2015). Caracterização do cenário econômico para os próximos 10 anos (2015-2024). https://www.epe.gov.br/sitespt/publicacoes-dados-abertos/publicacoes/PublicacoesArquivos/publicacao-245/topico-264/DEA\%2012-15\%20NT\%20Cenario\%20economico\%2020152024vf\%5B1\%5D.pdf.

Fernandes, T. L., Lima, L. A., Tenório, L. X. S., Silva, M. L., \& Ghesti, G. F. (2017). Prospecção tecnológica: Uma visão das inovações e perspectivas do mercado cervejeiro. Cadernos de Prospecção, 10(4), 851-865. https://doi.org/10.9771/cp.v10i4.23039.

Food and Drug Administration. (2015). Color Additive Status List. U.S. Food and Drug Administration. https://www.fda.gov/industry/color-additiveinventories/color-additive-status-list.

Food Ingredients Brasil. (2009). Dossiê corantes: Corantes. Revista-Fi. https://revista-fi.com.br/upload_arquivos/201606/2016060213572001465326315.pdf.

Ghesti, G. F., Faria, B. S., Dias, F. R., Cunha Júnior, G. C., Amaral, H. L., Lima, L. A., Pires, L. G. S., Araújo, L. P., Tenório, L. X. S., Silva, M. L., \& Fernandes, T. L. (2016). Tutorial de busca nos principais bancos de patentes. Centro de Apoio ao Desenvolvimento Tecnológico. http://nic.cdt.unb.br/pdf/programaseprojetos/nupitec/LIVRO-BANCO\%20DE\%20PATENTES.compressed.pdf.

Grand View Research. (2017). Colorant market size worth $\$ 37.49$ billion by 2025. https://www.grandviewresearch.com/press-release/global-colorants-market.

INPI: Comissão Permanente de Propriedade Intelectual. http://www.cppi.ufv.br/pt-BR/inpi.

Meinicke, R. M. (2008). Estudo da produção de pigmentos por Monascus ruber CCT 3802 utilizando glicerol como substrato em cultivo submerso. Dissertação de mestrado, Universidade Federal de Santa Catarina, Florianópolis, SC, Brasil.

Oliveira, C. F. D., Costa, J. P. V., \& Vendruscolo, F. (2019). Maltose syrup residue as the substrate for Monascus pigments production. Biocatalysis and Agricultural Biotechnology, 18, 1-8. https://doi.org/10.1016/j.bcab.2019.101101.

Pereira, A. S., Shitsuka, D. M., Pereira, F. J. \& Shitsuka, R. (2018). Metodologia da pesquisa científica. UFSM. https://repositorio.ufsm.br/bitstream/handle/1 /15824/Lic_Computacao_Metodologia-Pesquisa-Cientifica.pdf?sequence=1.

Prado, M. A. \& Godoy, H. T. (2003). Corantes artificiais em alimentos. Alimentos e Nutrição Araraquara, 14 (2), 237-250.

Rêgo, T. F., Correia, A. M. M., Araújo, J. A. R., Gurgel, J. C. \& Souza, A. (2015). Expectativas de retorno e riscos percebidos no agronegócio corante natural de caju para o mercado B2B. Custos e @ gronegócio on line. 11 (2), 370-400.

Ribeiro, N. M. (2018). Prospecção Tecnológica. Coleção PROFNIT. IFBA. 